Check for updates

Cite this: RSC Adv., 2019, 9, 30773

\title{
A straightforward synthesis of phenyl boronic acid (PBA) containing BODIPY dyes: new functional and modular fluorescent tools for the tethering of the glycan domain of antibodies $\dagger$
}

\author{
Gina Elena Giacomazzo, \$ Pasquale Palladino, D \& Cristina Gellini, \\ Gianluca Salerno, Veronica Baldoneschi, Alessandro Feis, (D) Simona Scarano, \\ Maria Minunni (D) and Barbara Richichi (D)*
}

\begin{abstract}
Received 31st July 2019
Accepted 24th September 2019

DOI: 10.1039/c9ra07608e

rsc.li/rsc-advances
\end{abstract}

\begin{abstract}
We report here on the efficient and straightforward synthesis of a series of modular and functional PBABODIPY dyes 1-4. They are an outstanding example of the efficient merge of the versatility of the 3,5dichloro-BODIPY derivatives and the receptor-like ability of the PBA moiety. The potential bioanalytical applicability of these tools was assessed by measuring the binding to glycan chains of antibodies by a Quartz Crystal Microbalance (QCM).
\end{abstract}

High performance organic fluorophores (e.g. large molar extinction coefficient, high quantum yield, high photo-stability, large Stokes shift) represent a booming research topic, with a wide range of applications ranging from materials to life sciences. ${ }^{1,2}$ In this framework, modular and functional probes have become highly sought-after and researchers' attention is manly focused on the fine tuning of optical properties and new functionalities for highly versatile tethering. However, despite the huge amount of effort in this field, only few fluorophores meet all these theoretical claims. ${ }^{3}$ Moreover, the availability of convenient and few steps synthetic protocols associated with a good overall yield is one of the major bottlenecks that needs to be addressed for ensuring the successful wide applicability of these probes. ${ }^{4} \mathrm{~A}$ brilliant example of a versatile fluorophore is the UV-absorbing dye 4,4'-difluoro-4-bora-3a,4a-diaza- $s$-indacene, known as BODIPY, ${ }^{5}$ which displays high quantum yield, tunable photo-physical properties and excellent photostability. ${ }^{5,6}$ Strategic structural modifications of BODIPY's molecular architecture offer an unparalleled opportunity to tune its spectroscopic features, and diverse successful functional and bioactive BODIPY-like probes have been proposed and some commercialized, so far. ${ }^{7}$ 3,5-Dichloro-BODIPY dyes are one of the most recent synthetic analogues whose potential versatility has been demonstrated by Dehaen and Boens. ${ }^{8}$ They

Department of Chemistry 'Ugo Schiff', University of Florence, Via Della Lastruccia 13, Sesto Fiorentino, 50019 FI, Italy. E-mail: barbara.richichi@unifi.it

$\dagger$ Electronic supplementary information (ESI) available: Protocols for the synthesis of compounds 1-8, fully characterization of compounds 1-8, selected NMR spectra of compounds 1-8. Scalar fluorescence and absorbance spectra, molar extinction coefficients in diverse media of compounds 1-4. Protocols for QCM and QY measurements. See DOI: 10.1039/c9ra07608e

‡ G. G. and P. P. equally contributed to this work. showed that appropriate substituents at 3,5 positions of the pyrrole shifted the excitation/emission bands of the corresponding substituted BODIPY derivatives. Thus, the 3,5dichloro-BODIPY dyes give access, by nucleophilic substitution, to a variety of symmetrical and non-symmetrical BODIPY with several applications including labeling, sensing, energy transfer cassette. ${ }^{\mathbf{e}, \mathbf{8 , 9}}$ Then, 3,5-dichloro-BODIPY derivatives have been directly employed for the selective detection, in in vitro models, of sulphur containing metabolites. ${ }^{10}$ Recently, the valuable properties of other BODIPY dyes have been used for saccharides detection by phenyl boronic acid (PBA) moiety introduced at the meso position of the BODIPY core. ${ }^{11}$ Notably, the dynamic covalent interaction between boronic acid and saccharides has been studied since the pioneering work of Lorand et al. ${ }^{12}$ and PBA ability to bind 1,2 and 1,3-cis-diols motifs of carbohydrates has been used for the development of synthetic 'boron-lectins' ${ }^{13}$ and lately for the fishing of glycoproteins from complex mixtures, for the site-oriented immobilization of antibodies and for biorthogonal conjugations. ${ }^{14}$ However, the few examples of PBA-BODIPY probes have been reported to date ${ }^{\mathbf{1 1}}$ and they are confined to 3,5-dimethyl-BODIPY derivatives that require specific protocols for the further functionalization.

In this context, we report here a straightforward synthetic route to obtain the functional and modular PBA-containing dye $\mathbf{1}$ and the subsequent late-stage diversification of the architecture of 1. Therefore, we obtained a small family of PBA-BODIPY derivatives with a 'traffic light' emission range (Fig. 1) for which we have studied the optical properties in different solvents and in physiological media. Thus, the present approach aims at the outstanding merging of the versatility of the 3,5-dichloroBODIPY dyes with the presence of a functional PBA at the meso position of the BODIPY core. Finally, we show an example 

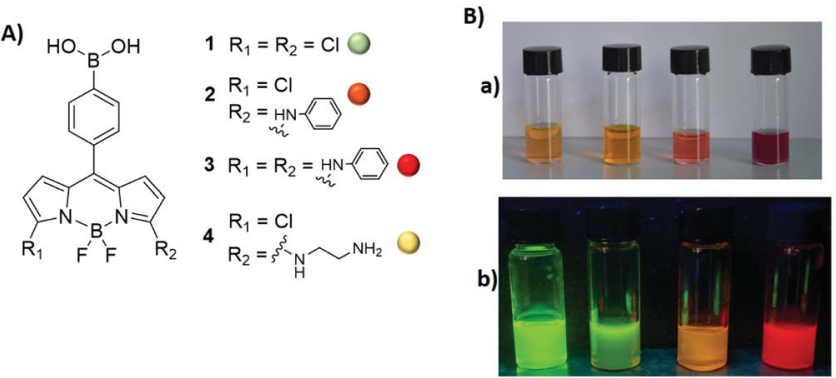

Fig. 1 (A) Structure of PBA-BODIPY derivatives 1-4. (B) Solution $\left(1.0 \mathrm{mg} \mathrm{mL}^{-1}\right.$ in $\mathrm{MeOH}$ ) of PBA-BODIPY derivatives (from the left to the right PDA-BODIPY $1,4,2,3)(\mathrm{a})$ under white light and (b) under UVlight $\left(\lambda_{\mathrm{ex}}=364 \mathrm{~nm}\right)$.

of the formation of the boronate esters between the PBABODIPY 4 and the glycan chains of an antibody, i.e. antistreptavidin monoclonal antibody, by using Quartz Crystal Microbalance.

The BODIPY-core is traditionally accessible following a few steps synthetic strategy, ${ }^{\mathbf{8 a}, \mathbf{1 5}}$ however, the main concerns are related to the low overall yields of some synthetic steps and to the small scale availability of BODIPY derivatives due to the unstable nature of pyrrole derivatives and/or of some synthetic intermediates. ${ }^{15 a, 16}$ In this paper, the boron dipyrromethane dye 1 (Scheme 1) was prepared following the synthetic strategy usually reported for the synthesis of 3,5-dichloro-BODIPY dyes ${ }^{15}$ by using the commercially available pyrrole-based derivatives and $p$-substituted benzaldehyde as starting materials.

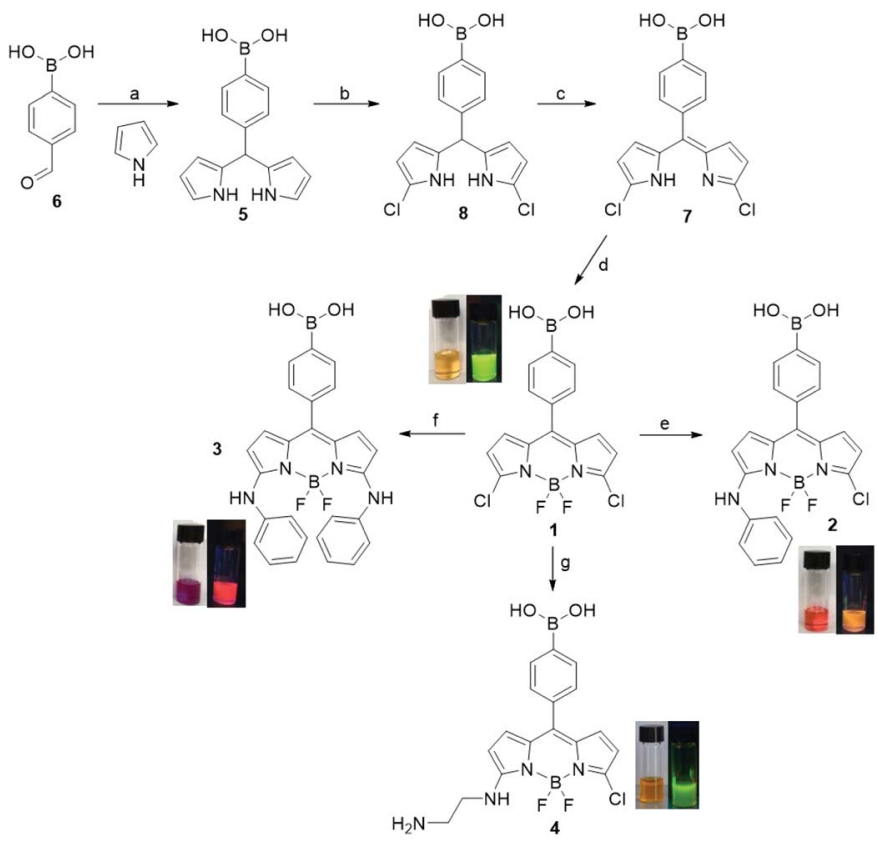

Scheme 1 Synthesis of the PBA-BODIPY $1-4$. (a) $\mathrm{CF}_{3} \mathrm{COOH}, 0{ }^{\circ} \mathrm{C} \rightarrow$ r.t., 15 minutes, $74 \%$; (b) NCS, THF, $-78{ }^{\circ} \mathrm{C} \rightarrow-20{ }^{\circ} \mathrm{C}, 18$ h; (c) DDQ, $\mathrm{CH}_{2} \mathrm{Cl}_{2}$, r.t., $16 \mathrm{~h}, 75 \%$ over two steps; (d) $\mathrm{BF}_{3} \mathrm{Et}_{2} \mathrm{O}, \mathrm{CH}_{2} \mathrm{Cl}_{2}$, r.t., 2 h, $75 \%$; (e) aniline, $\mathrm{CH}_{2} \mathrm{Cl}_{2}, 60{ }^{\circ} \mathrm{C}, 48 \mathrm{~h}, 58 \%$; (f) aniline, $140{ }^{\circ} \mathrm{C}, 0.5 \mathrm{~h}$; (g) ethylendiamine, r.t., 45 minutes, $44 \%$.
At first, the meso-PBA substitute dipyrromethane $\mathbf{5}$ was prepared by acid-catalyzed condensation ${ }^{\mathbf{8 a , 1 5 a , b}}$ of commercially available 4-formylbenzeneboronic acid 6 with neat excess (25 eq.) pyrrole (Scheme 1). In general, the purification of dipyrromethane derivatives is not trivial, ${ }^{\mathbf{1 5 a}, \boldsymbol{b}, \mathbf{1 6}}$ depending on substituents at the meso position, as previously reported..$^{\mathbf{8}, \mathbf{1 5 b}}$ A mixture of side products, have been identified and fully characterized, ${ }^{\mathbf{1 5 b}}$ and some improvements on the purification steps have been reported $^{15 a, b}$ switching from flash chromatography to a bulb-tobulb distillation followed by recrystallization protocols (yield 27-68\%). ${ }^{15 b}$ Here, we set up, a straightforward crystallization protocol for the isolation of the pure dipyrromethane 5 (74\%) avoiding the low yielding flash chromatography and tedious bulb-to-bulb distillations elsewhere described. ${ }^{15 b}$ We have also examined the effect related to the workup of the reaction media (pyrrole : 6, 25:1 ratio, catalytic TFA) on the yield of this synthetic step, by using diverse protocols to refine the formation of 5, observing an higher yield (74\% vs. 50\%) and an easier removal of side products, ${ }^{15 b}$ by the addition of trimethylamine (see ESI $\dagger$ ) to neutralize the TFA, and the subsequent concentration to dryness of the reaction mixture. Notably, the use of pure 5 is critical for the overall yield of the two following synthetic steps. The chlorination of 5 (Scheme 1) with $N$-chlorosuccinimide (NCS) followed by oxidation with 2,3-dichloro-5,6dicyano- $p$-benzoquinone (DDQ) afforded the 3,5-disubstututed dipyrromethene 7 (75\% over two steps). Finally, 7 was reacted with boron trifluoride diethyl etherate $\left(\mathrm{BF}_{3}-\mathrm{OEt}_{2}\right)$ and $N, N^{\prime}-$ diisopropylethylamine (DIPEA) to give the green emitting PBABODIPY 1 (75\%). Dye 1 is amenable to structural modifications at the 3,5 positions of the pyrrole moiety so we prepared a small family of dyes which showed the spectral shift in the absorption and emission bands depending on the substitution pattern (Scheme 1). To demonstrate the versatility of PBABODIPY 1 we selected aniline as nucleophile already studied in this kind of reactions. ${ }^{\boldsymbol{9} \boldsymbol{e}_{\boldsymbol{f}}}$ Thus, the PBA-BODIPY 1 was reacted with an excess of aniline in dichloromethane to give PBABODIPY 2 (58\%) as pink solid, according to earlier investigations showing that one of the two chlorine atoms is more prone to be involved in the nucleophilic substitution. ${ }^{\mathbf{8}, 9}$ Therefore, the di-substitute PBA-BODIPY 3 has been obtained from 3,5dichloro-BODIPY $\mathbf{1}$ by using more stringent experimental conditions (neat excess of aniline, $140{ }^{\circ} \mathrm{C}$ ) as previous reported. ${ }^{9 f}$ Finally, mono-substitute PBA-BODIPY 4 was prepared in order to have a functional group on the BODIPY-core useful for further functionalization of the dye for the following studies. Thus, 1 was reacted with a neat excess of 1,2-diaminoethane to afford the yellow emitting PBA-BODIPY 4 (44\%).

The spectroscopic properties of the PBA-BODIPY dyes 1-4 (Fig. 2, and S1-S11 $\dagger$ ) were studied in methanol, dichloromethane, water and physiological PBS buffer $(\mathrm{pH}=7.4)$. The spectra reported in the left side of Fig. 2, show the absorption pattern of known PBA-BODIPY derivatives 1-4. ${ }^{\mathbf{8 , 9 , 1 7}}$ In all tested media, the main absorption band attributed to the $0-0$ band of the strong $\mathrm{S}_{0} \rightarrow \mathrm{S}_{1}$ transition is located around $510 \mathrm{~nm}$ for PBABODIPY 1 and 2 (Fig. 2A, and C) and $590 \mathrm{~nm}$ for PBA-BODIPY 3 (Fig. 2E). For PBA-BODIPY 4 (Fig. 2G) the $S_{0} \rightarrow S_{1}$ transition is not the main absorption and is observed around $490 \mathrm{~nm}$. The 

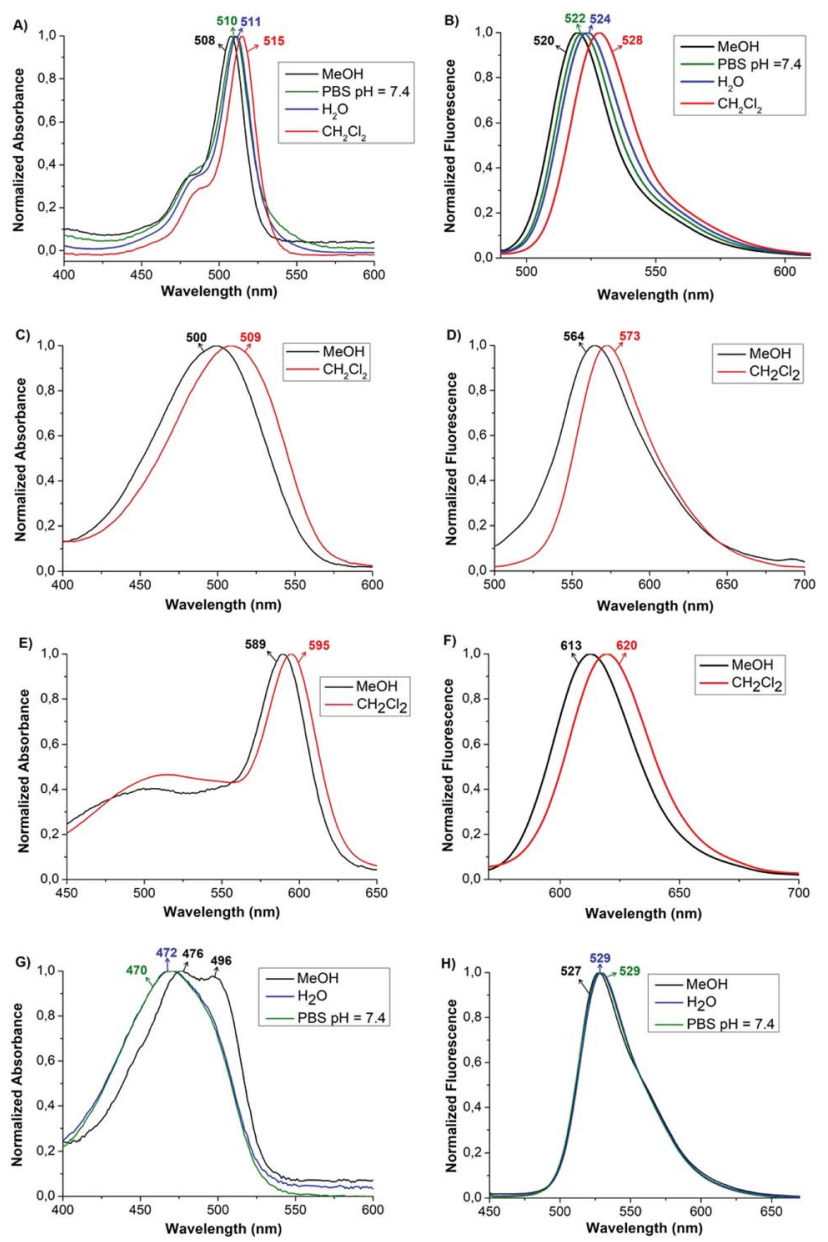

Fig. 2 Normalized absorbance and fluorescence spectra of PBABODIPY 1-4 in $\mathrm{MeOH}$ (black line) physiological PBS buffer $\mathrm{pH}=7.4$ (green line), $\mathrm{H}_{2} \mathrm{O}$ (blue line) and $\mathrm{CH}_{2} \mathrm{Cl}_{2}$ (red line). (A and $\mathrm{B}$ ) PBABODIPY 1; (C and D) PBA-BODIPY 2; ( $E$ and F) PBA-BODIPY 3; ( $G$ and H) PBA-BODIPY 4.

data are summarized in Table 1 . The shoulders at shorter wavelengths for the symmetric dyes 1 and 3, at $\sim 480 \mathrm{~nm}$ and $\sim 515 \mathrm{~nm}$ respectively, have been assigned to the $0-1$ vibronic

Table 1 Absorption and fluorescence emission spectral data of PBABODIPY 1-4

\begin{tabular}{llclccc}
\hline DYE & Solvent & $\lambda_{\text {abs }}(\mathrm{nm})$ & $\lambda_{\mathrm{em}}(\mathrm{nm})$ & $\Delta \tilde{\nu}\left(\mathrm{cm}^{-1}\right)$ & $\Phi_{\mathrm{f}}$ & $\varepsilon$ \\
\hline 1 & $\mathrm{MeOH}$ & 508 & 520 & 454 & 0.16 & $55389 \pm 246$ \\
& $\mathrm{PBS}$ & 510 & 522 & 451 & & $31453 \pm 405$ \\
& $\mathrm{H}_{2} \mathrm{O}$ & 511 & 524 & 485 & & $39011 \pm 355$ \\
& $\mathrm{CH}_{2} \mathrm{Cl}_{2}$ & 515 & 528 & 478 & & $46873 \pm 395$ \\
2 & $\mathrm{MeOH}^{2}$ & 500 & 564 & 2270 & 0.0048 & $29008 \pm 146$ \\
& $\mathrm{CH}_{2} \mathrm{Cl}_{2}$ & 509 & 573 & 2194 & & $28701 \pm 249$ \\
3 & $\mathrm{MeOH}^{4}$ & 589 & 613 & 665 & 0.22 & $13315 \pm 62$ \\
4 & $\mathrm{CH}_{2} \mathrm{Cl}$ & 595 & 620 & 678 & & $27043 \pm 520$ \\
& $\mathrm{MeOH}$ & 496 & 527 & 1186 & 0.017 & $21454 \pm 77$ \\
& & 476 & & & & \\
& $\mathrm{PBS}$ & $\sim 494$ & 529 & 1340 & & $16810 \pm 94$ \\
& & 470 & & & & \\
& $\mathrm{H}_{2} \mathrm{O}$ & $\sim 493$ & 529 & 1381 & & $17912 \pm 132$
\end{tabular}

band of the same transition. For PBA-BODIPY 4 the $0-1$ vibronic transition is observed at $476 \mathrm{~nm}$ in $\mathrm{MeOH}$. In the right side of Fig. 2, the normalized fluorescence spectra of PBA-BODIPY 1-4 are also reported. A reduced solvatochromic effect is observed as both the $S_{0} \rightarrow S_{1}$ absorption and the emission bands are slightly red-shifted in dichloromethane than in more polar media (e.g. $\mathrm{MeOH}$ or $\mathrm{H}_{2} \mathrm{O}$ ). The broader full width at half maximum as well as the shape of the absorption and fluorescence bands of asymmetric PBA-BODIPY dyes 2 and 4 are consistent with previously reported amino-containing dyes and they were attributed to the diverse contributes of the proposed Lewis structural formulas. ${ }^{9 b}$ The molar extinction coefficient $(\varepsilon)$ was assessed in the four different solvents, showing that the highest hyperchromic effect is observed in $\mathrm{MeOH}$ (Table 1, Fig. S1-S11†). Finally, the Stokes shift values result moderately low for the symmetric derivatives $\mathbf{1}$ and $\mathbf{3}$, while higher value are found for dyes 2 and 4 , where symmetry is reduced. The $S_{1} \rightarrow S_{0}$ fluorescence quantum yields $\left(\Phi_{\mathrm{f}}\right)$ of 1-4 have been measured in MeOH using Rhodamine 6G as standard reference for 1, 2 and 4 and DODCl for 3 (see ESI $\dagger$ ) in the same solvent. High values of $\Phi_{\mathrm{f}}$ have been found for 1 and 3, 0.16 and 0.22 respectively, suggesting that the symmetric presence of bulky substituents on the indacene moiety can stiffen the molecule, promoting radiative decay processes towards the ground state. Moreover, the relatively small Stokes shift observed for both compounds point at a molecular structure that is not much affected by the electronic excitation. On the opposite, the large Stokes shifts measured for the dyes 2 and $\mathbf{4}$, indicate a non negligible molecular structure variations going from $S_{0}$ to $S_{1}$. The less rigid skeleton of these dyes can endorse thermal relaxation, lowering the respective $\Phi_{\mathrm{f}}$, as shown in Table 1 .

It is well-established that boronic acids can form fast and reversible covalent interactions with 1,2/1,3 cis-diols of carbohydrates generally affording five-/six-membered cyclic boronic esters. ${ }^{13}$ In this paper, as case sample for the proof of the interaction between our PBA-BODIPY dyes and the glycan unit of glycosylated proteins using Quartz Crystal Microbalance (QCM) for gravimetric sensing. $N$-Glycosylation site of the Fc regions of monoclonal antibodies (mAbs) have attached significant attention in the last year as site end-on attachment of mAbs in diverse bioanalytical assays, ${ }^{\mathbf{1 4 c}, \boldsymbol{d}}$ and for the purification of mAbs from cell mixtures. ${ }^{14 e}$ Sialic acid (SA) has been claimed to be the anchoring point of such interactions, ${ }^{14 c}$ however the site of PBA-SA interaction is still high debated topic and comprehensive studies combining DFT calculations and NMR spectroscopy have been published. ${ }^{17}$ Here the binding of rabbit IgG was assessed by monitoring the resonance frequency decrease on $9.5 \mathrm{MHz}$ crystals induced by mass increase on the surface carrying the boronic acid derivative 4 (Fig. 3A).

The gold electrodes sandwiching the quartz crystals were modified as showed in Fig. 3. Quartz crystals were exposed to a $1 \mathrm{mg} \mathrm{\textrm {L } ^ { - 1 }}$ solution of a non-glycosylated protein (namely, streptavidin, SAv) in $20 \mathrm{mM}$ Hepes $\mathrm{pH} \mathrm{8.5,} \mathrm{that} \mathrm{was} \mathrm{proved} \mathrm{to}$ minimize secondary interactions, ${ }^{14 c}$ allowing to exclude nonspecific binding of SAv to 4 (data not shown). Subsequently, the crystals were exposed to a $1 \mathrm{mg} \mathrm{L}^{-1}$ solution of IgG in the same buffer. Such experiment tested the coupling of 

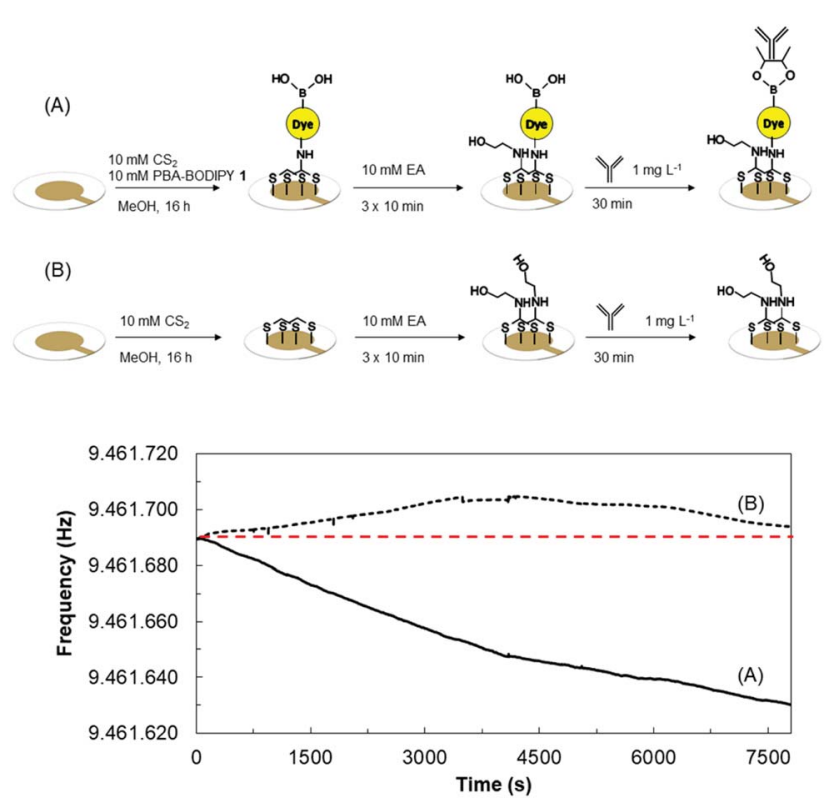

Fig. 3 QCM experimental set up, where crystal 1 (A) was functionalized with 4, while crystal 2 (B) was kept as the negative control. The online QCM sensorgrams shows the resonance frequency shift occurred after antibody deposition on crystal 1 black solid line, (A) and crystal 2 (black dotted line, (B)).

glycosylated portion of the mAb to the boronic acid functions of 4. The surfaces were then rinsed with buffer to remove nonspecifically bound antibody. Fig. 3 shows the recorded frequency shifts after IgG interaction and baseline equilibration, about $60 \mathrm{~Hz}$ for positive control (A) and $1 \mathrm{~Hz}$ for negative control (B). Thus, confirming that the antibody was specifically bound to the crystal functionalized with the boronic acid, likely through the glycosylated portions.

In summary, we reported here an efficient methodology for the synthesis of PBA-BODIPY dyes and we demonstrated their tunability in terms of optical properties and capability in tethering of glycans, appearing instrumental and reliable tools for diverse bio-related research fields.

\section{Conflicts of interest}

There are no conflicts to declare.

\section{Acknowledgements}

We thank MIUR-Italy ("Progetto Dipartimenti di Eccellenza 2018-2022" allocated to Department of Chemistry "Ugo Schiff"). We thank Dr Annalisa Guerri from the Department of Chemistry, University of Florence (IT) for technical assistance.

\section{Notes and references}

1 (a) Y. Fu and N. S. Finney, RSC Adv., 2018, 51, 29051; (b) K.-N. Wang, X.-J. Chao, B. Liu, D.-J. Zhou, L. He, X.-H. Zheng, Q. Cao, C.-P. Tan, C. Zhang and Z.-W. Mao, Chem. Commun., 2018, 54, 2635; (c) P. Gao, W. Pan, N. Li and B. Tang, Chem. Sci., 2019, 10, 6035; (d) K. Sharma, V. Sharma and S. S. Sharma, Nanoscale Res. Lett., 2018, 13, 381; (e) V. Lakshmi, M. R. Rao and M. Ravikanth, Org. Biomol. Chem., 2015, 13, 2501.

2 (a) L. D Lavis and R. T. Raines, ACS Chem. Biol., 2008, 3, 142 and references cited therein; (b) M. A. Reppy, J. Fluoresc., 2008, 18, 461; (c) P. Palladino, V. Castelletto, A. Dehsorkhi, D. Stetsenko and I. W. Hamley, Chem. Commun., 2012, 48, 9774.

3 (a) X. Li, X. Gao, W. Shi and H. Ma, Chem. Rev., 2014, 114, 590; (b) J. O. Escobedo, O. Rusin, S. Lim and R. M. Strongin, Curr. Opin. Chem. Biol., 2010, 14, 64.

4 B. Tang, F. Lv, K. Chen, L. Jiao, Q. Liu, H. Wang and E. Hao, Chem. Commun., 2019, 55, 4691.

5 (a) A. Treibs and F. H. Kreuzer, Justus Liebigs Ann. Chem., 1968, 718, 208; (b) L. Jean-Gérard, W. Vasseur, F. Scherninski and B. Andrioletti, Chem. Commun., 2018, 54, 12914.

6 (a) A. Loudet and K. Burgess, Chem. Rev., 2007, 107, 4891; (b) H. Kowada and K. Kikuchi, Chem. Soc. Rev., 2015, 44, 4953; (c) N. Boens, V. Leen and W. Dehaen, Chem. Soc. Rev., 2012, 41, 1130; (d) Y. Ni and J. Wu, Org. Biomol. Chem., 2014, 12, 3774.

7 (a) H. Lu, J. Mack, Y. Yang and Z. Shen, Chem. Soc. Rev., 2014, 43, 4778; (b) G. Ulrich, R. Ziessel and A. Harriman, Angew. Chem., Int. Ed., 2008, 47, 1184; (c) N. Boens, B. Verbelen and W. Dehaen, Eur. J. Org. Chem., 2015, 6577; (d) K. Krumova and G. Cosa, J. Am. Chem. Soc., 2010, 132, 17560; (e) S. H. Son, S. Daikoku, A. Ohtake, K. Suzuki, K. Kabayama, Y. Ito and O. Kanie, Chem. Commun., 2014, 50, 3010.

8 (a) M. Baruah, W. Qin, N. Basarić, W. M. De Borggraeve and N. Boens, J. Org. Chem., 2005, 70, 4152; (b) T. Rohand, M. Baruah, W. Qin, N. Boens and W. Dehaen, Chem. Commun., 2006, 266; (c) S. Yin, V. Leen, S. Van Snick, N. Boens and W. Dehaen, Chem. Commun., 2010, 46, 6329; (d) W. Qin, T. Rohand, M. Baruah, A. Stefan, M. Van der Auweraer, W. Dehaen and N. Boens, Chem. Phys. Lett., 2006, 420, 562; (e) S. Yin, V. Leen, C. Jackers, D. Beljonne, B. Van Averbeke, M. Van der Auweraer, N. Boens and W. Dehaen, Chem.-Eur. J., 2011, 17, 13247; (f) W. Qin, V. Leen, T. Rohand, W. Dehaen, P. Dedecker, M. Van der Auweraer, K. Robeyns, L. Van Meervelt, D. Beljonne, B. Van Averbeke, J. N. Clifford, K. Driesen, K. Binnemans and N. Boens, J. Phys. Chem. A, 2009, 113, 439; $(g)$ W. Qin, V. Leen, W. Dehaen, J. Cui, C. Xu, X. Tang, W. Liu, T. Rohand, D. Beljonne, B. Van Averbeke, J. N. Clifford, K. Driesen, K. Binnemans, M. Van der Auweraer and N. Boens, J. Phys. Chem. A, 2009, 113, 11731.

9 (a) J. C. Er, M. K. Tang, C. G. Chia, H. Liew, M. Vendrell and Y.-T. Chang, Chem. Sci., 2013, 4, 2168; (b) Y. A. Volkova, B. Brizet, P. D. Harvey, A. D. Averin, C. Goze and F. Denat, Eur. J. Org. Chem., 2013, 4270; (c) D. W. Domaille, L. Zeng and C. J. Chang, J. Am. Chem. Soc., 2010, 132, 1194; (d) J. Han, O. Gonzalez, A. Anguilar-Aguilar, E. Pena-Cabrera and K. Burgess, Org. Biomol. Chem., 2009, 7, 34; (e) N. Dorh, S. Zhu, K. B. Dhungana, R. Pato, F.-T. Luo, H. Liu 
and A. Tiwari, Sci. Rep., 2015, 5, 18337; (f) E. Fron, E. CoutiñoGonzalez, L. Pandey, M. Sliwa, M. Van der Auweraer, F. C. De Schryver, J. Thomas, Z. Dong, V. Leen, M. Smet, W. Dehaen and T. Vosch, New J. Chem., 2009, 33, 1490; $(g)$ T. Rohand, J. Lycoops, S. Smout, E. Braeken, M. Sliwa, M. Van der Auweraer, W. Dehaen, W. M. De Borggraeve and N. Boens, Photochem. Photobiol. Sci., 2007, 6, 1061; (h) L. Li, B. Nguyen and K. Burgess, Bioorg. Med. Chem. Lett., 2008, 18, 3112.

10 L. Y. Niu, Y. S. Guan, Y. Z. Chen, L. Z. Wu, C. H. Tung and Q. Z. Yang, J. Am. Chem. Soc., 2012, 134, 18928.

11 (a) B. Liu, N. Novikova, M. C. Simpson, M. S. M. Timmer, B. L. Stocker, T. Sohnel, D. C. Ware and P. J. Brothers, Org. Biomol. Chem., 2016, 14, 5205; (b) J. Zhai, T. Pan, J. Zhu, Y. Xu, J. Chen, Y. Xie and Y. Qin, Anal. Chem., 2012, 84, 10214; (c) J. S. Hansen, J. F. Petersen, T. Hoeg-Jensen and J. B. Christensen, Tetrahedron Lett., 2012, 53, 5852; (d) J. S. Hansen, T. Hoeg-Jensen and J. B. Christensen, Tetrahedron, 2017, 73, 3010; (e) N. DiCesare and J. B. Lakowicz, Tetrahedron Lett., 2001, 42, 9105.

12 J. P. Lorand, J. Org. Chem., 1959, 24, 769.

13 (a) X. Wu, X.-X. Chen and Y.-B. Jiang, Analyst, 2017, 142, 1403; (b) X. Wu, Z. Li, X.-X. Chen, J. S. Fossey, T. D. James and Y.-B. Jiang, Chem. Soc. Rev., 2013, 42, 8032; (c) X.-T. Zhang, G.-J. Liu, Z.-w. Ning and G.-w. Xing, Carbohydr. Res., 2017, 452, 129; (d) A. Matsumoro and Y. Miyahara, Sci. Technol. Adv. Mater., 2018, 19, 18.
14 (a) B. Akgun and D. G. Hall, Angew. Chem., Int. Ed., 2016, 55, 3909; (b) A. Bandyopadhyay and J. Gao, J. Am. Chem. Soc., 2016, 138, 2098; (c) F. Duval, T. A. van Beek and H. Zuilhof, Analyst, 2015, 140, 6467; (d) H. Y. Song, J. Hobley, X. Su and X. Zhou, Plasmonics, 2014, 9, 851; (e) A. M. Azavedo, A. G. Gomes, L. Borlido, I. F. S. Santos, D. M. F. Prazeres and M. R. Aires-Barros, J. Mol. Recognit., 2010, 23, 569.

15 (a) C.-H. Lee and J. S. Lindsey, Tetrahedron, 1994, 50, 11427; (b) B. J. Litter, M. A. Miller, C.-H. Hung, R. W. Wagner, D. F. O'Shea, P. D. Boyle and J. S. Lindsey, J. Org. Chem., 1999, 64, 1391; (c) M. R. Rao, S. M. Mobin and M. Ravikanth, Tetrahedron, 2010, 66, 1728.

16 C. Bruckner, V. Karunaratne, S. J. Rettig and D. Dolphin, Can. J. Chem., 1996, 74, 2182.

17 (a) J. A. Peters, Coord. Chem. Rev., 2014, 268, 1; (b) S. Nishitani, Y. Maekawa and T. Sakata, ChemistryOpen, 2018, 7, 513; (c) I. R. Vlahov, P. I. Vlahova and R. J. Linhardt, J. Am. Chem. Soc., 1997, 119, 1480; (d) H. Otsuka, E. Uchimura, H. Koshino and T. Okano, J. Am. Chem. Soc., 2003, 125, 3495; (e) K. Djanashvili, L. Frullano and J. A. Peters, Chem.-Eur. J., 2005, 11, 4010; (f) H. Otsuka, E. Uchimura, H. Koshino, T. Okano and K. Kataoka, J. Am. Chem. Soc., 2003, 125, 3493; $(g)$ M. Regueiro-Figueroa, K. Djanashvili, D. Esteban-Gòmez, A. de Blas, C. Platas-Iglesias and T. Rodrìguez-Blas, Eur. J. Org. Chem., 2010, 3237. 